FROM THE THIRD GENEVA CONFERENCE ON PERSON-CENTERED MEDICINE: CULTURAL DIVERSITY AND PERSON-CENTERED HEALTH CARE

\title{
Person-centered Care and Economic Deprivation: an epidemiological view of positive mental health in a developing country
}

\author{
Javier E. Saavedra MD DR MdSc ${ }^{\mathrm{ab}}$, Victor Cruz MD ${ }^{\mathrm{ab}}$, Juan E. Mezzich MD MA MSc PhD ${ }^{\mathrm{c}}$ \\ a Instituto Nacional de Salud Mental, "Honorio Delgado-Hideyo Noguchi", Lima, Peru \\ b Universidad Peruana Cayetano Heredia, Departamento de Psiquiatría y Salud Mental, Lima, Peru \\ c Deputy Editor-in-Chief, International Journal of Person Centered Medicine, Professor of Psychiatry, Mount \\ Sinai School of Medicine, New York University, USA, President, International Network for Person-centered \\ Medicine
}

\begin{abstract}
Positive health is one of the main components of person centered integrative diagnosis, as it involves the understanding of the patient's clinical condition within his context. Poverty is an important contextual factor that has been related to negative aspects of mental health in many studies, but has been studied little in regard to positive health, raising the importance of integrative care in poor persons. Objective: To identify in an adult population the extent to which poverty indicators are related to positive mental health indicators. Methods: The study consisted of a face-to-face household survey of 6555 community aged 18 years and older residents in five cities of the coast of Peru, selected trough a probabilistic three-stage sample procedure. The study integrated person-centered diagnosis proposals and other positive aspects of health as part of a comprehensive assessment which included an adaptation and complementation of the International Guidelines for Diagnostic Assessment (IGDA) diagnostic suggestions through the following instruments: the MINI International Neuropsychiatric Interview ICD-10 version, the Quality of Life Index, the Mental Health Questionnaire (MHQ) elaborated in Colombia. Socioeconomic data were assessed using a question from the MHQ about the family capacity to supply essential needs with the household income. Relational statistical analyses between the different components of the diagnostic formulation and other positive aspects of health and socioeconomic condition were conducted. Results: The average age was 39 years, illiteracy rate was 3.6\%; 59.2\% of the sample had not worked in the last week and the poverty line measured by the family capacity to supply essential needs with the household income was $25.5 \%$. Economic deprivation was associated with the worst indicators of positive mental health such as lower scores on quality of life measures, more selfcare functioning difficulties, lower satisfaction with personal aspects such as physical appearance, intelligence, education or work conditions, less feelings of happiness, more perceived psychosocial stressors with work, children and health and lower family integration. Conclusions: The implications of socioeconomic aspects in regard to the comprehensive diagnostic processes, treatment and research should be considered, particularly in less-developed countries. Health policies based on person-centered care programs, through the assessment of positive health, could help services to be more sensitive to more vulnerable sectors of the populations.
\end{abstract}

\section{Keywords}

Person-centered care, poverty, positive health, mental health, developing countries, epidemiology, quality of life, personal satisfaction

\section{Correspondence Address}

Dr. Javier E. Saavedra, Oficina Ejecutiva de Apoyo a la Investigación y Docencia Especializada, Instituto Nacional de Salud Mental, “Honorio Delgado - Hideyo Noguchi”, Jr. Eloy Espinoza Saldaña 709, San Martin de Porras, Lima-31, Peru, E-mail: saavedra@terra.com.pe.

Accepted for publication: 13 February 2011 


\section{Introduction}

An extensive body of literature supports the contention that the patient-centered approach to medical care, in its understanding of the patients' point of view, their health problems and circumstances, is fundamental to effective health care [1, 2]. Many scholars and clinicians have called for the development of a moral epistemology directed towards the achievement of a full integration of facts and values, the latter related to considering the patients social and psychological realms [3]. Themes associated with centeredness in health care reported in the literature have been associated with respect for individuality and values, therapeutic alliance, attention to social contexts and relationships, the integration of health and well-being, active participation and responsibility in the formulation of the treatment plan, sensitive and interactional communication, autonomy and the involvement of the professional as a person [4]. In this context, a personcentered integrative diagnosis has been proposed in line with the impending revision of the International Classification of Diseases ICD-11 [5]. This proposal involves broadening the patient's diagnosis to encompass the overall health status of the person presenting for care, covering both ill-health (or disease) and positive health.

Positive health is, in fact, one of the main components of the person-centered approach and is related to many aspects of health which concern the individual and the person. Positive health has been defined as the space between pathology or illness and the totality of health and dimensions identified in the literature have been related to adaptive functioning (in regard to personal care, occupational functioning, functioning with the family and social functioning), environmental and personal factors contextual to clinical problems, personal resources, emotional and instrumental social supports and quality of life [6]. Other aspects that have been linked to positive health are satisfaction with life, feelings of happiness, subjective well-being, sense of personal control, resilience and coping (related to functioning), sense of coherence, and optimism [7]. The combined consideration of positive and negative aspects of illness anticipates the emergence of rigorous empiric investigations toward the formulation of a science of well-being [8] and the prevention of illness and promotion of health to a higher level, as much as in the clinical environment as in that of the public health $[9,10,11]$.

Antecedents of approaches to an integral diagnosis that not only involves pathological aspects, but also positive ones, could be found in multiaxial outlines such as the one proposed by Ramirez in 1989 [12] and the Cuban Glossary of Psychiatry in the year 2000 [13]. The first one proposed 8 axes that included abilities and talents, spirituality and characterologic maturity. Also, the official inclusion in the DSM-IV of a cultural formulation, that took into consideration the individual's cultural identity, context and his culture, as a narrative complement to the standard diagnostic formulation, served as preamble to new integrative proposals [14].

Recently, the World Psychiatric Association (WPA) has considered a more comprehensive approach to diagnosis through two diagnostic levels. The first one is a standardized multiaxial diagnosis that describes the patient's illness and the patient's clinical condition through typologies and standardized scales. The second one is an idiographic diagnostic formulation that supplements the standardized formulation with personalized and flexible statements $[15,16]$. In the same way, the Section of Classification and Diagnostic of the Latin American Psychiatric Association in developing the Latin American Guide of Diagnostic Psychiatric (GLADP) has suggested the inclusion of both formulations in all diagnostic activity of the specialty [17].

Nevertheless, Corin et al have pointed out that the starting point of medical care is no longer located only in the individual, but in the constant interaction between the person and its environment [18]. Here, persons in poverty configure a special group at risk for unfavorable health conditions and inequities [19]. Poverty is one of the main problems in developing countries; in Latin America 33\% of the population live in poverty and $12.9 \%$ live in extreme poverty [20]. Poor persons are confronted with many contextual factors and negative environments such as unsuitable housing conditions, overcrowding, unemployment or unsuitable employment conditions which create a detrimental environment for mental health and which may easily translate into stress, anxiety, depression and despair, although some investigators believe that this is an indirect effect dependent upon the circumstances related to poverty [21]. This situation not only increases mental health problems in marginal sectors, but also jeopardizes their possibilities of accessing the appropriate services and care [22]. Such circumstances emphasize the importance of an integrative and person-centered approach when encountering persons with health problems. Poverty has been related to negative aspects of mental health in many studies, but fewer studies have investigated its impact on positive health, which raises the importance of integrative care in poor persons. Much less work has been done in developing countries where the use of income as a measure socioeconomic status could yield some methodological problems because of the considerable proportion of persons who do not have regular incomes. Person-centered medicine, by focusing on the person as a whole and emphasizing contextual factors, is of great importance in such conditions of economic deprivation. In order to emphasize the importance of the person-centered approach in this group of vulnerable persons, the exploration of positive mental health status should be considered of considerable significance.

The main objective of this study was to examine the extent of the relationship between poverty and variables related to positive mental health. This study contributes to the existing studies in different ways. It uses a representative sample from a developing country and it 
considers different aspects of positive health that are assessment suggestions for a person-centered approach, such as quality of life, problems with functioning, satisfaction with personal aspects, feelings of happiness, work satisfaction and family issues.

\section{Methods}

The present study is based on data from the 2006 Epidemiological Study of Mental Health in the Peruvian Coast (ESMHPC) [23]. This face to face survey was conducted by the Peruvian National Institute of Mental Health (PNIMH) between July 2006 and December 2006 and provided population estimates of mental disorders and other mental health indicators from 5 cities in the coast of Peru. The total population of these cities was 3,130,380 inhabitants at the time of the survey. The survey sampling frame was based on the information provided by the National Institute of Statistics and Data Processing (INEI) according to the Pre-survey of 1999. The sample was selected trough a probabilistic three-stage procedure: (1) area segments of clusters of households, (2) household units and (3) respondents. Individuals included in the sample were household residents who lived either permanently or regularly in private houses. Collective housing facilities such as hospitals, hotels, army headquarters, convents, etc., were excluded from the study. The study involved the selection of four units of analysis on each household: an adolescent aged between 12 and 17; an adult 18 year or older; an older adult aged 60 or older; and a married or cohabitant woman, head of the family or united with the head of the family. A Kish table was used to select each one unit of analysis, except for the married or cohabitant woman. The survey required written informed consent from respondents and was approved by the Institutional Ethics Committee. The survey was conducted by health professionals, mostly psychologists, who undertook 70 hours of training. The training involved: instruction about the survey content, practice sessions and practice field trainings in non-selected households in the community. A psychological evaluation of the interviewers was included to assure the suitability of persons for interviewing. A monitoring system which consisted of random household supervisions, re-interviews and witnessing interviews by a supervisor, concordance analysis supervisor-interviewers, quantitative and qualitative analyses and other measures, were implemented to ensure data quality. The initially selected number of sampling household was 7,020 for the adults, adolescents and married or cohabitant woman and 9,360 for the older adults. The participation level was $93.9 \%$ for the adults, $95.6 \%$ for the adolescents, $92.3 \%$ for the older adults and $94.9 \%$ for the married or cohabitant woman. The adult final sample consisted of 6,555 respondents, which is the sample used in this study.

\section{Measures}

In previous studies conducted by the PNIMH, all instruments use in this survey were subject to a pilot trial of field interviews and individual focus groups, which facilitated a thorough review of all documents and led to the final adjustments of the questions to be used in the surveys $[24,25,26,27]$. The statistical validity and reliability of the instruments used by the PNIMH has been recently published [28]. The core ESMHCP questions related to this study derived from the Mental Health Questionnaire (MHQ) elaborated in Colombia [29], the Quality of Life Index (QLI) [30], and questions related to five areas of functioning or disabilities elaborated with the guidelines of the WHO DAS-S [31] and the IGDA [15].

Mental Health Questionnaire. The MHQ adapted in Peru [32] gathers information of many mental health areas related to positive health pertinent to the person-centered approach. Questions selected for the present study include the following topics:

a. Feelings of happiness (1-item). Participants were asked: "How frequently do you feel happy?" The response was given on a 5-point scale, "never", "seldom”, “occasionally”, "almost always” and "always”. For statistical purposes and for gaining more power analyses, three outputs were used: "Never or seldom”, "Occasionally” and "Always or almost always”.

b. Personal satisfaction (9-items). Participants were asked, "How satisfied or pleased do you feel with your... "physical appearance”, “skin color", "intelligence", "socioeconomic condition", "the profession or trade you studied”, "level of education achieved", "religion”, “friendships or social relationships" and "place of residence". Each item was rated via a 5-point scale: "none”, "little”, “more or less”, “a lot”, “quite a lot”. The internal consistency for these questions was 0.791 according to the Chronbach's alpha model; questions grouped together in only one dimension [28]. For statistical purposes and for gaining more power analyses the following three outputs were used: "None or little", "More or less" and "A lot or quite a lot".

c. Family relationships (6-items). Participants were asked 6 questions regarding their family relationships. Questions included, "How much would you care to be a disgrace to your family?”, How much do you feel they respect you?", "How much do you feel that you support each other?”, "How proud do you feel of your family?, "How much do you feel your family's moral values are similar to your own", "How much do you feel you are part of your family's progress?. The response was given on each item via a 5-point scale: "none”, "little”, “more or less”, “a lot”, “quite a lot”. The internal consistency for these questions was 0.767 according to the Chronbach's alpha model [28]. For statistical purposes and for gaining more power 
analyses, the following three outputs were used: "None or little", "More or less" and "A lot or quite a lot”.

d. Presence of psychosocial stressors (7-items). Participants were asked, "How much tension or problems do the following situations caused you?” ... "your work or studies", "your children or other relatives”, “your partner”, "your money”, "your health", "law issues" and "other problems". The response was given on each item via a 5-point scale"none”, "little”, “more or less”, “a lot”, “quite a lot”. The internal consistency for these questions was 0.757 according to the Chronbach's alpha model; questions grouped together in only one dimension [28]. For statistical purposes and for gaining more power analyses, the following three outputs were used: "None or little", "More or less" and "A lot or quite a lot”.

e. Presence of environmental stressors (5-items). Participants were asked, "In the environment where you live, how much are you disturbed by... "noise", "the lack of ventilation", "the lack of space", "smell" and "other". The response was given on each item via a 5-point scale: “none”, "little”, “more or less”, “a lot", "quite a lot". The internal consistency for these questions was 0.785 according to the Chronbach's alpha model [28]. For this study "The garbage" was added as another environmental stressor. For statistical purposes and for gaining more power analyses, the following three outputs were used: "None or little", "More or less” and “A lot or quite a lot”.

The Quality of Life Index is a 10-item scale developed to assess aspects related to the construct of quality of life. Each item is rated from 1 to 10 . It has been validated in a Peruvian sample, showing adequate reliability and validity. [33] Items refer to physical wellbeing, psychological or emotional wellbeing, self-care and independent functioning, interpersonal functioning, social and emotional support, communitarian and services support, personal realization, spiritual satisfaction and global quality of life. The internal consistency for this scale found in the epidemiological studies of the PNIMH was 0.807 according to the Chronbach's alpha model [28].

Disabilities or functioning. Questions related to functioning or disabilities consisted of the four areas suggested by WHO DAS-S [34] and the IGDA [15]: selfcare, occupational functioning, family functioning, and broad social functioning. A fifth item related to organizational functioning was added. Interviewers responded to the following questions, "Do you have difficulties or limitations to regularly maintaining your personal hygiene, apparel or clothing?”, “Do you have difficulties or limitations in fulfilling efficiently your occupations (studies, work or home)?”, “Do you have difficulties or limitations in fulfilling satisfactorily your duties as mother, father, spouse or son (daughter)?, "Do you have difficulties or limitations in relating to relatives, friends, neighbors or persons in general?”, “Do you have difficulties or limitations in planning your activities, organizing and executing them?” Each item was rated on a 5-point scale: “none”, “minimal”, “moderate”, "severe”, "total or absolute". The internal consistency for these questions was 0.901 according to the Chronbach's alpha model; questions grouped together in only one dimension [28]. For statistical purposes and for gaining more power analyses, were conducted using two outputs: "None" or "At least some".

Demographic and socioeconomic data. Sociodemographic data included were age, gender, place of birth, native language, marital status and employment status. The latter considered those who were working the previous week and those looking for a job. The criterion used to define poverty regards a questions from the MHQ, which involves a simple subjective question posed to the wife or the family head, "Would you say that, usually, your family income is sufficient?”. The response was given via four possible answers, that for our study represents four levels of socioeconomic status: "It does not even meet the basic needs for food", "It meets only the basic need for food, but not others" "It meets only the basic needs for food, clothing, household, education and transport, but not others", "It meets the basic needs and also others like hobbies or entertainment". Families who were unable to meet their essential need for food were considered "extremely poor"; the families that could only cover their nutritional needs, but no other basic needs such as health, education, housing, etc. were qualified as "poor"; the families that were able to meet their basic needs, but no other needs (such as entertainment, recreation, differentiated education) were considered "just not poor" and those who were able to meet their basic needs and other needs were classified as "not poor".

\section{Analyses}

All estimate prevalences were weighted to account for the known probability of selection as well as to restore the distribution of the population unless otherwise stated. The analysis was conducted by means procedures without replacement for non-respondents. The cities were used to define strata. To test the main questions of the study for poverty status, linear regressions were calculated to examine the association between household income and poverty levels for use in this study. Contrast statements were utilized to determine whether the mean household income from each of the 4 levels of poverty differed significantly from each other. Relational statistical analyses were conducted between socioeconomic condition or poverty level and some components of the diagnostic formulation suggested by IGDA [15] (psychosocial stressors, adaptive functioning and quality of life) and 
Table 1. Sociodemographic characteristics of total population in five cities of the coast of Peru (n=6,555)

\begin{tabular}{|c|c|}
\hline & $\begin{array}{c}\text { Mean } \\
(95 \% \mathrm{Cl})\end{array}$ \\
\hline Weighted average age (standard error) & 39.4 (SE 0.37)(38.6-40.1) \\
\hline Age Group & $\%(95 \% \mathrm{Cl})$ \\
\hline 18 to 24 years & $24.3(22.0-26.7)$ \\
\hline 25 to 44 years & $40.4(37.7 .2-43.1)$ \\
\hline 45 to 64 years & $25.2(23.4-27.0)$ \\
\hline $65+$ & $10.2(8.9-11.6)$ \\
\hline \multicolumn{2}{|l|}{ Sex } \\
\hline Female & 50.1 (47.8-53.3)55.0 (unweighted) \\
\hline \multicolumn{2}{|l|}{ Educational Level } \\
\hline Illiteracy & $3.6(2.9-4.4)$ \\
\hline None & $2.4(1.9-3.0)$ \\
\hline Pre-primary education / Kindergarten & $0.1(0.0-0.2)$ \\
\hline Primary education & $16.2(14.1-18.6)$ \\
\hline Secondary education & $41.0(38.9-43.2)$ \\
\hline Baccalaureate & $0.1(0.0-0.2)$ \\
\hline Post secondary non-tertiary education & $15.0(13.5-16.7)$ \\
\hline Tertiary education & $24.2(21.9-26.6)$ \\
\hline \multicolumn{2}{|l|}{ Marital status } \\
\hline Cohabitation & $19.4(17.1-22.0)$ \\
\hline Separated & $7.2(6.4-8.1)$ \\
\hline Divorced & $0.7(0.5-1.1)$ \\
\hline Widowed & $4.9(4.2-5.7)$ \\
\hline Married & $35.1(33.4-36.8)$ \\
\hline Single & $32.7(30.2-35.2)$ \\
\hline \multicolumn{2}{|l|}{ Employment Conditions } \\
\hline Worked the previous week & $59.2(56.5-61.9)$ \\
\hline Looking for a job & $3.5(2.6-4.7)$ \\
\hline \multicolumn{2}{|c|}{ Level of Poverty According to Self-Perceived Coverage of Basic Needs with Family Income } \\
\hline Do not even meet basic need of food (Extremely poor) & $0.9(0.7-1.3)$ \\
\hline Do meet only the basic need of food, but not other basic needs (Poor) & $24.6(22.0-27.3)$ \\
\hline Do meet all basic needs but not other needs (just not poor) & $65.7(63.1-68.2)$ \\
\hline Do meet basic needs and other needs like hobbies or entertainment (Not poor) & $8.8(7.4-10.5)$ \\
\hline
\end{tabular}

$\mathrm{Cl}=$ Confidence Interval; SE= Standard error

other positive aspects of health. First, the general characteristics of the sample were analyzed, such as: average age, age groups, distribution by sex, occupation, education, socioeconomic status according to income and level of poverty. Here, descriptive statistical analyses were carried out such as frequency and main tendency measures and percentages were estimated. Prevalence rates were obtained on the distributions of positive mental health variables at the time of the interview. Next, bivariate analyses were carried out with the aim of identifying associations between the poverty variables and a number of variables such as age, sex, level of education, occupation, level of poverty, income level, and area of residence. If the independent variable was nominal (sex, level of education achieved, poverty etc.), chi square tests converted to the F statistic were carried out to consider the design of the survey (through the statistical package SPSSV13), taking into account a significance level of $<0.05$. Linear regressions were calculated to examine the association between poverty levels and quality of life scores. Contrast statements were utilized to determine whether the mean quality of life score from each of the three lower levels of socioeconomic status differed significantly from the highest level.

\section{Results}

\section{Sociodemographics}

A total of 6,555 adults were interviewed. The sample was distributed $45 \%$ males and $55 \%$ were females. The average age was 39 years (95\% CI, 38.6-40.1), illiteracy rate was $3.6 \%$ (95\% CI; 2.9-4.4) 59.2\% (95\% CI, 56.5-61.9) of the sample were not working last week and almost a quarter of the sample was considered poor measured by the selfperceived coverage of basic needs with household income. Only $0.9 \%$ (95\% CI, 0.7 - 1.3) of the sample were considered extremely poor. Significant differences were found between poverty status and marital status, particularly, a higher frequency of separate, divorced or widowed were found in the extremely poor group (30.5\% vs $8.7 \%$ ) (Table 2). Poverty status was also significantly associated with level of education and illiteracy; poorer individuals have less education as compared to not poor persons. All groups presented low occupancy (59.1\% $56.0 \%)$ frequency, and no statistically differences was found between them. No statistically differences in regard 
Table 2. Sociodemographic characteristics in the population in five cities of the coast of Peru $(n=6,555)$ according to the Perceived Coverage of Basic Needs with Family

\begin{tabular}{|c|c|c|c|c|c|c|c|c|c|c|c|}
\hline \multirow{2}{*}{\multicolumn{2}{|c|}{ SOCIODEMOGRAPHICS }} & \multicolumn{2}{|c|}{$\begin{array}{c}\text { f Basic Needs with Fam } \\
\left({ }^{*}\right) \\
\text { Prevalences \% } \\
(95 \% \mathrm{Cl})\end{array}$} & \multicolumn{4}{|c|}{ Overall Test } & \multirow[b]{2}{*}{$\mathrm{p}$} & \multicolumn{2}{|c|}{ Prevalence Ratio } & \multirow[b]{2}{*}{$\mathrm{A} / \mathrm{D}$} \\
\hline & & B & $\mathrm{C}$ & $\mathrm{D}$ & $F$ & df1 & df2 & & $A / B$ & $A / C$ & \\
\hline AGE, $Y$ & & & & & 0.757 & 5.555 & 3222 & 0.594 & & & \\
\hline $18-24$ & $\begin{array}{c}21.1 \\
(15.6-27.9)\end{array}$ & $\begin{array}{c}24.9 \\
(22.3-27.7)\end{array}$ & $\begin{array}{c}24.2 \\
(20.4-28.4)\end{array}$ & $\begin{array}{c}17.8 \\
(7.4-37.0)\end{array}$ & & & & & 0.85 & 0.87 & 1.19 \\
\hline $25-44$ & $\begin{array}{c}46.0 \\
(39.1-53.0)\end{array}$ & $\begin{array}{c}40.4 \\
(37.5-43.3)\end{array}$ & $\begin{array}{c}38.5 \\
(32.8-44.6)\end{array}$ & $\begin{array}{c}35.5 \\
(22.9-50.4)\end{array}$ & & & & & 1.14 & 1.19 & 1.30 \\
\hline $45-64$ & $\begin{array}{c}23.1 \\
(17.7-29.4)\end{array}$ & $\begin{array}{c}25.2 \\
(23.2-27.3)\end{array}$ & $\begin{array}{c}25.3 \\
(21.8-29.2)\end{array}$ & $\begin{array}{c}35.6 \\
(21.8-52.2)\end{array}$ & & & & & 0.92 & 0.91 & 0.65 \\
\hline $65+$ & $\begin{array}{c}9.8 \\
(5.2-17.8)\end{array}$ & $\begin{array}{c}9.5 \\
(7.5-11.9)\end{array}$ & $\begin{array}{c}12.0 \\
(9.8-14.6)\end{array}$ & $\begin{array}{l}11.2 \\
(6.0-19.9)\end{array}$ & & & & & 1.03 & 0.82 & 0.88 \\
\hline SEX & & & & & 1.850 & 2.321 & 1346 & 0.151 & & & \\
\hline Male & $\begin{array}{c}48.9 \\
(41.0-56.8)\end{array}$ & $\begin{array}{c}49.2 \\
(46.4-52.0)\end{array}$ & $\begin{array}{c}51.5 \\
(48.1-54.9)\end{array}$ & $\begin{array}{c}69.9 \\
(57.8-79.8)\end{array}$ & & & & & 0.99 & 0.95 & 0.70 \\
\hline Female & $\begin{array}{c}51.1 \\
(43.2-59.0)\end{array}$ & $\begin{array}{c}50.8 \\
(48.0-53.6)\end{array}$ & $\begin{array}{c}48.5 \\
(45.1-51.9)\end{array}$ & $\begin{array}{c}30.1 \\
(20.2-42.2)\end{array}$ & & & & & 1.01 & 1.05 & 1.70 \\
\hline MARITAL STATUS & & & & & 4.684 & 6.449 & 3740 & 0.00006 & & & \\
\hline Cohabitant & $\begin{array}{c}15.5 \\
(8.9-25.5)\end{array}$ & $\begin{array}{c}18.0 \\
(15.9-20.4)\end{array}$ & $\begin{array}{c}24.7 \\
(21.3-28.6)\end{array}$ & $\begin{array}{c}17.9 \\
(10.3-29.3)\end{array}$ & & & & & 0.86 & 0.63 & 0.87 \\
\hline $\begin{array}{l}\text { Separate, Divorced } \\
\text { or widowed }\end{array}$ & $\begin{array}{c}8.7 \\
(5.3-14.2)\end{array}$ & $\begin{array}{c}11.7 \\
(10.4-13.2)\end{array}$ & $\begin{array}{c}16.5 \\
(13.3-20.3)\end{array}$ & $\begin{array}{c}30.5 \\
(20.7-42.4)\end{array}$ & & & & & 0.74 & 0.53 & 0.29 \\
\hline Married & $\begin{array}{c}44.0 \\
(36.9-51.3)\end{array}$ & $\begin{array}{c}35.7 \\
(33.4-38.1)\end{array}$ & $\begin{array}{c}30.3 \\
(26.9-33.9)\end{array}$ & $\begin{array}{c}29.7 \\
(18.1-44.7)\end{array}$ & & & & & 1.23 & 1.45 & 1.48 \\
\hline Single & $\begin{array}{c}31.8 \\
(25.1-39.4)\end{array}$ & $\begin{array}{c}34.5 \\
(31.8-37.4)\end{array}$ & $\begin{array}{c}28.4 \\
(24.4-32.9)\end{array}$ & $\begin{array}{c}21.9 \\
(10.6-40.0)\end{array}$ & & & & & 0.92 & 1.12 & 1.45 \\
\hline \multicolumn{12}{|l|}{ OCCUPATION } \\
\hline $\begin{array}{ll}\text { Worked } & \text { the } \\
\text { previous week }\end{array}$ & $\begin{array}{c}56.0 \\
(47.6-64.1)\end{array}$ & $\begin{array}{c}59.7 \\
(56.7-62.6)\end{array}$ & $\begin{array}{c}59.3 \\
(54.9-63.5)\end{array}$ & $\begin{array}{c}59.1 \\
(45.0-71.8)\end{array}$ & 0.369 & 2.531 & 1468 & 0.741 & 0.94 & 0.94 & 0.95 \\
\hline Looking for job & $\begin{array}{c}2.2 \\
(1.0-4.7)\end{array}$ & $\begin{array}{c}3.3 \\
(2.1-5.3)\end{array}$ & $\begin{array}{c}4.4 \\
(3.2-6.1)\end{array}$ & $\begin{array}{c}5.7 \\
(2.2-14.0)\end{array}$ & 0.973 & 2.104 & 1220 & 0.382 & 0.67 & 0.50 & 0.39 \\
\hline \multicolumn{12}{|l|}{ EDUCATION } \\
\hline Level of education & & & & & 15.15 & 6.962 & 4038 & $<0.0001$ & & & \\
\hline No education & $\begin{array}{c}0.5 \\
(0.1-2.4)\end{array}$ & $\begin{array}{c}1.6 \\
(1.2-2.3)\end{array}$ & $\begin{array}{c}4.7 \\
(3.4-6.5)\end{array}$ & $\begin{array}{c}11.6 \\
(6.0-21.2)\end{array}$ & & & & & 0.31 & 0.11 & 0.04 \\
\hline Primary School & $\begin{array}{c}9.4 \\
(4.3-19.3)\end{array}$ & $\begin{array}{c}14.3 \\
(11.5-17.7)\end{array}$ & $\begin{array}{c}23.4 \\
(20.1-27.1)\end{array}$ & $\begin{array}{c}35.8 \\
(22.7-51.4)\end{array}$ & & & & & 0.66 & 0.40 & 0.26 \\
\hline Secondary School & $\begin{array}{c}24.6 \\
(19.2-31.0)\end{array}$ & $\begin{array}{c}40.2 \\
(37.3-43.2)\end{array}$ & $\begin{array}{c}49.7 \\
(44.3-55.1)\end{array}$ & $\begin{array}{c}31.9 \\
(18.4-49.4)\end{array}$ & & & & & 0.61 & 0.49 & 0.77 \\
\hline $\begin{array}{ll}\text { Superior } & \text { non } \\
\text { universitary } & \end{array}$ & $\begin{array}{c}20.5 \\
(15.8-26.2)\end{array}$ & $\begin{array}{c}15.2 \\
(13.4-17.3)\end{array}$ & $\begin{array}{c}12.4 \\
(10.1-15.0)\end{array}$ & $\begin{array}{c}14.4 \\
(6.4-29.1)\end{array}$ & & & & & 1.35 & 1.65 & 1.42 \\
\hline Superior University & $\begin{array}{c}45.0 \\
(38.3-51.8)\end{array}$ & $\begin{array}{c}28.6 \\
(25.9-31.5)\end{array}$ & $\begin{array}{c}9.8 \\
(7.5-12.7)\end{array}$ & $\begin{array}{c}6.3 \\
(2.0-18.3)\end{array}$ & & & & & 1.57 & 4.59 & 7.14 \\
\hline Illiteracy & $\begin{array}{c}0.2 \\
(0.0-0.7)\end{array}$ & $\begin{array}{c}2.3 \\
(1.7-3.1)\end{array}$ & $\begin{array}{c}7.8 \\
(5.9-10.3)\end{array}$ & $\begin{array}{c}14.5 \\
(7.4-26.3)\end{array}$ & 34.28 & 2.244 & 1301 & $<0.0001$ & 0.08 & 0.02 & 0.01 \\
\hline INCOME \$ & & & & & & & & & AvsB & BvsC & CvsD \\
\hline $\begin{array}{l}\text { Household income } \\
\text { (monthly av. in \$)+ } \\
(812.3, \quad 95 \% \quad \mathrm{Cl} \\
769.6-854.9 \%)\end{array}$ & $\begin{array}{c}456 \\
(407-505)\end{array}$ & $\begin{array}{c}283 \\
(270-297)\end{array}$ & $\begin{array}{c}177 \\
(165-188)\end{array}$ & $\begin{array}{c}137 \\
(103-171)\end{array}$ & 101.9 & 3 & 578 & $<0.0001$ & $<0.0001$ & $<0.0001$ & 0.028 \\
\hline
\end{tabular}

(*) $A=$ Cover the basic needs and other needs (Not poor); $B=$ Cover the basic needs but not others (Just not poor); C= Cover only basic nutritional needs (Poor); $D=$ Do not even cover the basic nutritional needs (Extremely Poor).

$(+)$ Exchange rate 1 US $\times 3$ NS

to socioeconomic status was found regarding to age and sex. Household income was significantly associated with the poverty measures use in the study and significant differences was found between each mean monthly income of the 4 poverty levels (Table 2 ).

\section{Feeling of happiness and personal satisfaction}

Significant differences were found between feelings of happiness and poverty levels $(\mathrm{p}=0.046)$. Feeling always or almost always happy decreases with the presence of 
Table 3. Prevalence of feelings of happiness and personal satisfaction in the population in five cities of the coast of Peru ( $n=6,555)$ according to the Perceived Coverage of Basic Needs with Family Income and Prevalence Ratio Among the Poor and Not Poor

\begin{tabular}{|c|c|c|c|c|c|c|c|c|c|c|c|}
\hline \multirow{2}{*}{$\begin{array}{l}\text { POSITIVE MENTAL } \\
\text { INDICATORS }\end{array}$} & \multicolumn{4}{|c|}{$\begin{array}{l}\text { Coverage of Basic Needs with Family Income } \\
\qquad\left({ }^{*}\right)\end{array}$} & \multicolumn{3}{|c|}{ Independence Test } & \multicolumn{4}{|c|}{ Prevalence Ratio } \\
\hline & & $(95$ & $\left.{ }^{\circ} \mathrm{Cl}\right)$ & & \multirow[b]{2}{*}{$\mathrm{F}$} & \multirow[b]{2}{*}{ df1 } & \multirow[b]{2}{*}{ df2 } & \multirow[b]{2}{*}{$\mathrm{p}$} & \multirow[b]{2}{*}{ A/B } & \multirow[b]{2}{*}{$\mathrm{A} / \mathrm{C}$} & \multirow[b]{2}{*}{ A/D } \\
\hline $\begin{array}{l}\text { Prevalences } \\
\text { (General Population ) }\end{array}$ & $\begin{array}{c}A \\
N=501\end{array}$ & $\begin{array}{l}B \\
N=4157\end{array}$ & $\begin{array}{c}C \\
N=1802\end{array}$ & $\begin{array}{c}D \\
N=88\end{array}$ & & & & & & & \\
\hline $\begin{array}{l}\text { Always or almost always } \\
(72.4 \%, 95 \% \mathrm{Cl} 70.4- \\
74.3 \%)\end{array}$ & $\begin{array}{c}85.8 \\
(81.2-89.5)\end{array}$ & $\begin{array}{c}73.6 \\
(71.4-75.6)\end{array}$ & $\begin{array}{c}65.0 \\
(60.1-69.5)\end{array}$ & $\begin{array}{c}55.7 \\
(42.5-68.2)\end{array}$ & 7.239 & 3.804 & 2207 & 0.00001 & 1.16 & 1.32 & 1.54 \\
\hline \multicolumn{12}{|c|}{ PERSONAL SATISFACTION (A LOT OR QUITE A LOT) } \\
\hline $\begin{array}{l}\text { Physical aspect (59.9\%, } \\
95 \% \mathrm{Cl} 57.1-62.5)\end{array}$ & $\begin{array}{c}65.3 \\
(57.8-72.1)\end{array}$ & $\begin{array}{c}61.4 \\
(59.0-63.7)\end{array}$ & $\begin{array}{c}54.3 \\
(48.9-59.6)\end{array}$ & $\begin{array}{c}44.7 \\
(31.8-58.3)\end{array}$ & 2.855 & 3.051 & 1769 & 0.035 & 1.06 & 1.20 & 1.46 \\
\hline $\begin{array}{l}\text { Skin color } \\
(76.3 \%, 95 \% \mathrm{Cl} 74.3- \\
78.3)\end{array}$ & $\begin{array}{c}81.5 \\
(72.7-88.0)\end{array}$ & $\begin{array}{c}78.5 \\
(76.2-80.7)\end{array}$ & $\begin{array}{c}69.1 \\
(64.4-73.5)\end{array}$ & $\begin{array}{c}65.3 \\
(50.3-77.8)\end{array}$ & 5.244 & 3.105 & 1800 & 0.001 & 1.04 & 1.18 & 1.25 \\
\hline $\begin{array}{l}\text { Intelligence }(70.1 \%, 95 \% \\
\mathrm{Cl} 67.7-72.3)\end{array}$ & $\begin{array}{c}75.1 \\
(67.3-81.5)\end{array}$ & $\begin{array}{c}72.6 \\
(69.6-75.3)\end{array}$ & $\begin{array}{c}62.1 \\
(56.6-67.3)\end{array}$ & $\begin{array}{c}56.5 \\
(43.9-68.2)\end{array}$ & 3.709 & 3.372 & 1956 & 0.008 & 1.03 & 1.21 & 1.33 \\
\hline $\begin{array}{l}\text { Socioeconomic status } \\
(26.1 \%, 95 \% \mathrm{Cl} 23.9- \\
28.5)\end{array}$ & $\begin{array}{c}46.1 \\
(37.7-54.8)\end{array}$ & $\begin{array}{c}27.2 \\
(24.6-30.0)\end{array}$ & $\begin{array}{c}16.6 \\
(14.2-19.4)\end{array}$ & $\begin{array}{c}11.5 \\
(4.8-25.0)\end{array}$ & 20.733 & 4.296 & 2491 & $<0.0001$ & 1.70 & 2.78 & 4.00 \\
\hline $\begin{array}{l}\text { Profession or trade } \\
\text { studied }(72.1 \%, 95 \% \mathrm{CI} \\
69.1-74.8)\end{array}$ & $\begin{array}{c}83.4 \\
(77.3-88.1)\end{array}$ & $\begin{array}{c}72.5 \\
(69.4-75.4)\end{array}$ & $\begin{array}{c}60.6 \\
(52.4-68.2)\end{array}$ & $\begin{array}{c}65.1 \\
(40.0-83.9)\end{array}$ & 6.898 & 4.847 & 2811 & $<0.0001$ & 1.15 & 1.38 & 1.28 \\
\hline $\begin{array}{l}\text { Level of achieved } \\
\text { education }(40.5 \%, 95 \% \\
\text { Cl } 37.8-43.3)\end{array}$ & $\begin{array}{c}56.3 \\
(48.8-63.5)\end{array}$ & $\begin{array}{c}43.0 \\
(39.9-46.1)\end{array}$ & $\begin{array}{c}28.5 \\
(24.2-33.2)\end{array}$ & $\begin{array}{c}32.3 \\
(19.3-48.8)\end{array}$ & 11.534 & 4.659 & 2702 & $<0.0001$ & 1.31 & 1.97 & 1.74 \\
\hline $\begin{array}{l}\text { Religion }(75.2 \%, 95 \% \mathrm{Cl} \\
72.6-77.6)\end{array}$ & $\begin{array}{c}78.1 \\
(69.3-85.0)\end{array}$ & $\begin{array}{c}75.3 \\
(72.6-77.8)\end{array}$ & $\begin{array}{c}73.8 \\
(70.4-77.0)\end{array}$ & $\begin{array}{c}74.6 \\
(62.4-83.8)\end{array}$ & 1.802 & 3.472 & 2013 & 0.135 & 1.04 & 1.06 & 1.05 \\
\hline $\begin{array}{l}\text { Friendships or social } \\
\text { relationships }(59.3 \% \text {, } \\
95 \% \text { IC 56.7-61.8) }\end{array}$ & $\begin{array}{c}70.8 \\
(62.3-78.0)\end{array}$ & $\begin{array}{c}59.8 \\
(56.7-62.9)\end{array}$ & $\begin{array}{c}54.2 \\
(49.6-58.7)\end{array}$ & $\begin{array}{c}44.2 \\
(31.5-57.7)\end{array}$ & 5.713 & 4.073 & 2362 & 0.00013 & 1.18 & 1.31 & 1.60 \\
\hline $\begin{array}{l}\text { Place of residence } \\
(55.9 \%, 95 \text { IC 52.9-58.8) }\end{array}$ & $\begin{array}{c}60.8 \\
(53.4-67.8)\end{array}$ & $\begin{array}{c}57.7 \\
(54.0-61.4)\end{array}$ & $\begin{array}{c}49.7 \\
(45.6-53.9)\end{array}$ & $\begin{array}{c}42.9 \\
(30.6-56.2)\end{array}$ & 3.377 & 4.153 & 2408 & 0.008 & 1.05 & 1.22 & 1.42 \\
\hline
\end{tabular}

$\left.{ }^{*}\right) \mathrm{A}=$ Cover the basic needs and other needs (Not poor); $\mathrm{B}=$ Cover the basic needs but not others (Just not poor); $\mathrm{C}=\mathrm{Cover}$ only basic nutritional needs (Poor); $\mathrm{D}=$ Do not even cover the basic nutritional needs (Extremely Poor).

poverty and differences were noticed between extremely poor individuals and each of the non poor groups (55.7\% vs $85.8 \%)$. High personal satisfaction on different aspect of life was lower in the poor groups as compared with the non poor group, except for religion. The relationship was significantly for physical aspect, skin color, intelligence, socioeconomic status, profession or trade studied, level of achieved education, social relationships and place of residence. Higher differences was found for satisfaction with socioeconomic status (11.5\% vs $46.1 \%)$, level of achieved education (32.3\% vs 56.3\%) and satisfaction with friendships or social relationships (44.2\% vs 70.8\%) (Table 3).

\section{Family relationships}

Compared to the "non poor" groups, the "poor" and "extremely poor" individuals showed significantly worse family indicators on all responses, "extremely poor" as well as "poor" individuals were significantly different from the "non poor" group. Higher differences were found for “feeling support between each other" (57.9\% vs 83.9\%), "feeling respect from family" (63.7\% vs 90.1\%) and "feeling part of the family progress" (57.8\% vs $81.6 \%)$ (Table 4).

\section{Contextual factors}

Significant differences were encountered between the presence of psychosocial stressors and poverty levels. A gradient of frequencies was found between groups, being higher for the "extremely poor" group and lower for the "not poor" group. The perception of "a lot" or "quite a lot"of tension in regard to work, children and relatives, money and health was higher in the group of poor individual compare with the groups of "not poor". Higher differences were found with children or other relatives (31.6\% vs $10.4 \%$ ) and money (55.5\% vs $19.0 \%)$. No significant differences between "poor" and "not poor" groups were found for law issues, studies and problems 
Table 4. Prevalence of family relationships indicators in the population in five cities of the coast of Peru $(n=6,555)$ according to the Perceived Coverage of Basic Needs with Family Income and Prevalence Ratio Among the Poor and Not Poor

\begin{tabular}{|c|c|c|c|c|c|c|c|c|c|c|c|}
\hline \multirow{3}{*}{$\begin{array}{c}\text { Prevalences of } \\
\text { positive mental health } \\
\text { indicators (General } \\
\text { Population) }\end{array}$} & \multicolumn{4}{|c|}{ Coverage of Basic Needs with Family Income $\left(^{*}\right)$} & \multirow{2}{*}{\multicolumn{4}{|c|}{ Independence Test }} & \multirow{2}{*}{\multicolumn{3}{|c|}{ Prevalence Ratio }} \\
\hline & \multicolumn{4}{|c|}{$\begin{array}{c}\% \\
(95 \% \mathrm{Cl})\end{array}$} & & & & & & & \\
\hline & A & B & C & D & \multirow[t]{2}{*}{$\mathbf{F}$} & \multirow[t]{2}{*}{ df1 } & \multirow[t]{2}{*}{ df2 } & \multirow[t]{2}{*}{$\mathbf{p}$} & \multirow[t]{2}{*}{ A/B } & \multirow[t]{2}{*}{$\mathrm{A} / \mathrm{C}$} & \multirow[t]{2}{*}{ A/D } \\
\hline & $N=501$ & $\mathrm{~N}=4157$ & $\mathrm{~N}=1802$ & $\mathrm{~N}=88$ & & & & & & & \\
\hline \multicolumn{12}{|c|}{ FAMILY COHESION (A LOT OR QUITE A LOT) } \\
\hline $\begin{array}{l}\text { Do care to be a } \\
\text { disgrace for family } \\
(87.4 \%, 95 \% \text { IC 85.1- } \\
89.3)\end{array}$ & $\begin{array}{c}92.0 \\
(88.3-94.6)\end{array}$ & $\begin{array}{c}88.6 \\
(86.0-90.9)\end{array}$ & $\begin{array}{c}82.6 \\
(78.8-85.8)\end{array}$ & $\begin{array}{c}75.0 \\
(58.8-86.3)\end{array}$ & 5.532 & 5.060 & 2935 & 0.00004 & 1.04 & 1.11 & 1.23 \\
\hline $\begin{array}{l}\text { Feeling respect from } \\
\text { family }(78.0 \%, 95 \% \text { IC } \\
76.5-79.5)\end{array}$ & $\begin{array}{c}90.1 \\
(85.8-93.1)\end{array}$ & $\begin{array}{c}78.5 \\
(76.8-80.2)\end{array}$ & $\begin{array}{c}72.8 \\
(69.2-76.1)\end{array}$ & $\begin{array}{c}63.7 \\
(50.7-75.0)\end{array}$ & 9.025 & 4.757 & 2759 & $<0.0001$ & 1.15 & 1.24 & 1.41 \\
\hline $\begin{array}{l}\text { Feeling support } \\
\text { between each other } \\
(73.8 \%, 95 \% \text { IC } 71.9 \text { - } \\
75.7)\end{array}$ & $\begin{array}{c}83.9 \\
(77.1-89.0)\end{array}$ & $\begin{array}{c}76.1 \\
(73.7-78.4)\end{array}$ & $\begin{array}{c}64.7 \\
(60.7-68.6)\end{array}$ & $\begin{array}{c}57.9 \\
(44.5-70.2)\end{array}$ & 11.103 & 3.865 & 2242 & $<0.0001$ & 1.10 & 1.30 & 1.45 \\
\hline $\begin{array}{l}\text { Feeling proud of family } \\
(86.4 \%, 95 \% \text { IC } 85.1- \\
87.6)\end{array}$ & $\begin{array}{c}93.2 \\
(89.5-95.6)\end{array}$ & $\begin{array}{c}88.3 \\
(86.7-89.7)\end{array}$ & $\begin{array}{c}79.5 \\
(75.8-82.7)\end{array}$ & $\begin{array}{c}70.1 \\
(58.3-79.7)\end{array}$ & 12.607 & 4.388 & 2545 & $<0.0001$ & 1.05 & 1.17 & 1.33 \\
\hline $\begin{array}{l}\text { Feeling family moral } \\
\text { values are similar to his } \\
\text { own }(70.7 \%, 95 \% \text { IC } \\
68.6-72.7)\end{array}$ & $\begin{array}{c}81.8 \\
(74.5-87.3)\end{array}$ & $\begin{array}{c}72.5 \\
(69.7-75.0)\end{array}$ & $\begin{array}{c}62.4 \\
(58.0-66.5)\end{array}$ & $\begin{array}{c}61.8 \\
(49.2-73.0)\end{array}$ & 6.360 & 3.640 & 2111 & 0.00008 & 1.16 & 1.31 & 1.32 \\
\hline $\begin{array}{l}\text { Feeling part of family } \\
\text { progress }(69.5 \%, 95 \% \\
\text { IC } 66.6-72.2)\end{array}$ & $\begin{array}{c}81.6 \\
(72.8-88.0)\end{array}$ & $\begin{array}{c}70.8 \\
(67.2-74.0)\end{array}$ & $\begin{array}{c}62.1 \\
(57.5-66.4)\end{array}$ & $\begin{array}{c}57.8 \\
(43.7-70.8)\end{array}$ & 5.151 & 3.355 & 1946 & 0.001 & 1.15 & 1.31 & 1.41 \\
\hline
\end{tabular}

with partner. In regard to environmental stressors, significant differences were found between "poor" individuals compared to "not poor" subjects for the presence of "a lot" or "quite a lot" of disturbances regarding smell, dust and garbage. The differences were significant only between basic "poor" individuals and "not poor" individuals. Some frequencies were higher for the group in extreme poverty but did not reach significance. No significant differences were found between "poor" and "non poor" groups for noise, ventilation, and lack of space between each group (Table 5).

\section{Quality of life}

Mean total QOLI scores and sub-scores decreased as the level of socioeconomic status decreased, indicating lower level of quality of life in the "poor" group as compared with the "not poor" groups. No significant difference was found in the total score between "poor" (mean = 7.18, SE= 0.05 ) and "extremely poor" groups (mean=6.81SE=0.20); and this was true for all sub-scores, except for global quality of life. However, significant differences were found between "extremely poor", "poor" and "just not poor" compared to the "not poor" group or highest level. The lowest QOLI scores in all groups were for communitarian support, where significant differences were found between the "poor group" (mean=5.53, SE=0.09) and the highest socioeconomic group (mean=6.12, $\mathrm{SE}=0.21$ ). The highest QOLI score were found for occupational functioning in the "not poor" group (mean=8.41, SE=0.09) (Table 6).

\section{Disabilities or functioning}

Higher frequencies of disability were found in all areas of functioning in the "poor" groups, but significant differences were found only in self-care functioning $(\mathrm{p}=0.002)$. In this area, significant differences were found between the "extremely poor" (8.6\%, 95\% CI 2.5-25.1) and "not poor" individuals (0.2\%, 95\% CI 0.0-0.6), as well as with the "just not poor" subjects (1.4\%, 95\% CI 0.9-.3). A tendency towards a significant difference between "poor" and "not poor" groups was found in regard to occupational functioning ( $\mathrm{p}=0.053)$ and family functioning (0.057) (Table 7). 
Table 5. Prevalence of Contextual Factors in the Population in five cities of the coast of Peru $(n=6,555)$ according to the Perceived Coverage of Basic Needs with Family Income and Prevalence Ratio Among the Poor and Not Poor

\begin{tabular}{|c|c|c|c|c|c|c|c|c|c|c|c|}
\hline \multirow{4}{*}{$\begin{array}{c}\text { Prevalences of } \\
\text { positive mental health } \\
\text { indicators (General } \\
\text { Population) }\end{array}$} & \multicolumn{4}{|c|}{ Coverage of Basic Needs with Family Income $\left({ }^{*}\right)$} & \multirow{2}{*}{\multicolumn{4}{|c|}{ Independence Test }} & \multirow{2}{*}{\multicolumn{3}{|c|}{ Prevalence Ratio }} \\
\hline & \multicolumn{4}{|c|}{$\begin{array}{c}\% \\
(95 \% \mathrm{Cl})\end{array}$} & & & & & & & \\
\hline & A & B & C & D & \multirow[t]{2}{*}{$\mathbf{F}$} & \multirow[t]{2}{*}{ df1 } & \multirow[t]{2}{*}{ df2 } & \multirow[t]{2}{*}{$\mathbf{p}$} & \multirow[t]{2}{*}{ B/A } & \multirow[t]{2}{*}{ CIA } & \multirow[t]{2}{*}{ DIA } \\
\hline & $\mathrm{N}=501$ & $\mathrm{~N}=4157$ & $\mathrm{~N}=1802$ & $N=88$ & & & & & & & \\
\hline \multicolumn{12}{|c|}{ PSYCHOSOCIAL STRESSORS ${ }^{\text {a }}$ (A LOT OR QUITE A LOT) } \\
\hline $\begin{array}{l}\text { Work }(24.4 \%, 95 \% \text { IC } \\
\text { 21.8-27.3) }\end{array}$ & $\begin{array}{c}19.6 \\
(13.9-26.8)\end{array}$ & $\begin{array}{c}22.3 \\
(19.3-25.6)\end{array}$ & $\begin{array}{c}30.9 \\
(24.1-38.5)\end{array}$ & $\begin{array}{c}45.0 \\
(28.1-63.1)\end{array}$ & 2.564 & 4.519 & 2621 & 0.030 & 1.14 & 1.58 & 2.30 \\
\hline $\begin{array}{l}\text { Studies }(18.7 \%, 95 \% \\
\text { IC } 15.3-22.7)\end{array}$ & $\begin{array}{c}11.8 \\
(6.9-19.5)\end{array}$ & $\begin{array}{c}19.5 \\
(15.4-24.4)\end{array}$ & $\begin{array}{c}20.2 \\
(12.2-31.4)\end{array}$ & $\begin{array}{c}40.8 \\
(5.8-88.5)\end{array}$ & 0.745 & 4.938 & 2864 & 0.588 & 1.65 & 1.71 & 3.46 \\
\hline $\begin{array}{l}\text { Children or other } \\
\text { relatives (21.4\%, } 95 \% \\
\text { IC 19.4-23.5) }\end{array}$ & $\begin{array}{c}10.4 \\
(7.4-14.5)\end{array}$ & $\begin{array}{c}19.8 \\
(17.4-22.4)\end{array}$ & $\begin{array}{c}29.2 \\
(25.6-33.1)\end{array}$ & $\begin{array}{c}31.6 \\
(19.8-46.4)\end{array}$ & 7.738 & 5.041 & 2924 & $<0.0001$ & 1.90 & 2.81 & 3.04 \\
\hline $\begin{array}{l}\text { Partner }(18.3 \%, 95 \% \\
\text { IC } 16.1-20.9)\end{array}$ & $\begin{array}{c}10.6 \\
(6.6-16.4)\end{array}$ & $\begin{array}{c}18.0 \\
(15.2-21.2)\end{array}$ & $\begin{array}{c}22.5 \\
(19.0-26.5)\end{array}$ & $\begin{array}{c}20.9 \\
(11.9-34.1)\end{array}$ & 2.324 & 4.100 & 2378 & 0.053 & 1.70 & 2.12 & 1.97 \\
\hline $\begin{array}{l}\text { Money }(32.6 \%, 95 \% \text { IC } \\
30.7-34.6)\end{array}$ & $\begin{array}{c}19.0 \\
(14.6-24.3)\end{array}$ & $\begin{array}{c}30.5 \\
(28.3-32.8)\end{array}$ & $\begin{array}{c}42.3 \\
(38.1-46.6)\end{array}$ & $\begin{array}{c}55.5 \\
(41.8-68.5)\end{array}$ & 12.523 & 4.953 & 2873 & $<0.0001$ & 1.61 & 2.23 & 2.92 \\
\hline $\begin{array}{l}\text { Health }(37.1 \%, 95 \% \text { IC } \\
35.0-39.2)\end{array}$ & $\begin{array}{c}33.0 \\
(26.8-39.8)\end{array}$ & $\begin{array}{c}35.0 \\
(32.3-37.8)\end{array}$ & $\begin{array}{c}43.4 \\
(39.6-47.3)\end{array}$ & $\begin{array}{c}57.0 \\
(42.0-70.9)\end{array}$ & 4.112 & 4.997 & 2898 & 0.001 & 1.06 & 1.32 & 1.73 \\
\hline $\begin{array}{l}\text { Law issues }(14.5 \% \text {, } \\
95 \% \text { IC 12.9-16.3) }\end{array}$ & $\begin{array}{c}10.7 \\
(7.0-15.9)\end{array}$ & $\begin{array}{c}14.9 \\
(12.8-17.2)\end{array}$ & $\begin{array}{c}14.5 \\
(12.1-17.4)\end{array}$ & $\begin{array}{c}22.8 \\
(12.1-38.9)\end{array}$ & 1.264 & 3.659 & 2122 & 0.283 & 1.39 & 1.36 & 2.13 \\
\hline \multicolumn{12}{|c|}{ ENVIRONMENTAL STRESSORS ${ }^{b}$ (A LOT OR QUITE A LOT) } \\
\hline $\begin{array}{l}\text { The Noise (22.9\%, } \\
95 \% \text { IC21.1\%-24.8\%) }\end{array}$ & $\begin{array}{c}22.6 \\
(17.4-28.8)\end{array}$ & $\begin{array}{c}22.6 \\
(20.3-25.1)\end{array}$ & $\begin{array}{c}23.7 \\
(20.3-27.5)\end{array}$ & $\begin{array}{c}22.8 \\
(10.8-41.9)\end{array}$ & 0.183 & 5.266 & 3054 & 0.973 & 1.00 & 1.05 & 1.01 \\
\hline $\begin{array}{l}\text { Lack of ventilation } \\
\text { (15.2\%, 95\% IC 13.6- } \\
\text { 16.9) }\end{array}$ & $\begin{array}{c}16.3 \\
(11.2-23.2)\end{array}$ & $\begin{array}{c}14.4 \\
(12.5-16.5)\end{array}$ & $\begin{array}{c}16.6 \\
(13.4-20.2)\end{array}$ & $\begin{array}{c}22.8 \\
(13.2-36.4)\end{array}$ & 1.059 & 4.551 & 2640 & 0.379 & 0.88 & 1.02 & 1.40 \\
\hline $\begin{array}{l}\text { Lack of space \%, } \\
\text { (20.4\%, 95\% IC 18.7- } \\
22.2)\end{array}$ & $\begin{array}{c}17.7 \\
(12.5-24.6)\end{array}$ & $\begin{array}{c}19.3 \\
(17.0-21.7)\end{array}$ & $\begin{array}{c}24.3 \\
(21.0-27.9)\end{array}$ & $\begin{array}{c}18.7 \\
(11.5-29.0)\end{array}$ & 1.729 & 4.412 & 2559 & 0.134 & 1.09 & 1.37 & 1.06 \\
\hline $\begin{array}{l}\text { The smell }(28.7 \%, 95 \% \\
\text { IC } 26.7 \%-30.7 \%)\end{array}$ & $\begin{array}{c}19.9 \\
(14.8-26.3)\end{array}$ & $\begin{array}{c}27.6 \\
(25.2-30.0)\end{array}$ & $\begin{array}{c}34.6 \\
(30.8-38.7)\end{array}$ & $\begin{array}{c}32.9 \\
(19.0-50.6)\end{array}$ & 3.346 & 4.895 & 2839 & 0.005 & 1.39 & 1.74 & 1.65 \\
\hline $\begin{array}{l}\text { The dust }(43.7 \%, 95 \% \\
\text { IC } 40.7 \%-46.7 \%)\end{array}$ & $\begin{array}{c}36.1 \\
(29.2-43.6)\end{array}$ & $\begin{array}{c}42.9 \\
(39.6-46.3)\end{array}$ & $\begin{array}{c}48.3 \\
(43.4-53.3)\end{array}$ & $\begin{array}{c}47.4 \\
(35.7-59.3)\end{array}$ & 3.289 & 4.417 & 2562 & 0.008 & 1.19 & 1.34 & 1.31 \\
\hline $\begin{array}{l}\text { The garbage }(51.7 \% \text {, } \\
95 \% \text { IC } 49.2-54.2)\end{array}$ & $\begin{array}{c}41.2 \\
(34.2-48.5)\end{array}$ & $\begin{array}{c}51.7 \\
(48.8-54.5)\end{array}$ & $\begin{array}{c}55.1 \\
(51.4-58.8)\end{array}$ & $\begin{array}{c}61.7 \\
(47.4-74.2)\end{array}$ & 4.114 & 5.146 & 2985 & 0.001 & 1.25 & 1.34 & 1.50 \\
\hline $\begin{array}{l}\text { (*) } \mathrm{A}=\text { Cover the basic } \\
\text { nutritional needs (Poor); } \\
\text { (a) Participants were as }\end{array}$ & ds and oth & needs (No & $\mathrm{n}$ do the $\mathrm{f}$ & the basic & eds $b$ & ot oth & & & & & \\
\hline
\end{tabular}


Table 6. Average quality of life in the population in five cities of the coast of Peru $(n=6,555)$ according to the Perceived Coverage of Basic Needs with Family Income and Prevalence Ratio Among the Poor and Not Poor

\begin{tabular}{|c|c|c|c|c|c|c|c|c|c|c|c|}
\hline \multirow{4}{*}{$\begin{array}{c}\text { Prevalences of } \\
\text { positive mental } \\
\text { health indicators } \\
\text { (General Population) }\end{array}$} & \multicolumn{4}{|c|}{ Coverage of Basic Needs with Family Income $\left(^{*}\right)$} & \multirow{2}{*}{\multicolumn{4}{|c|}{$\begin{array}{c}\text { Independence Test } \\
\text { overall }\end{array}$}} & & \multirow[b]{2}{*}{$\mathbf{p}$} & \multirow{4}{*}{$\mathrm{B} / \mathrm{A}$} \\
\hline & \multirow{3}{*}{$\begin{array}{c}\text { A } \\
N=501\end{array}$} & \multicolumn{2}{|c|}{$\begin{array}{l}\text { Average } \\
(95 \% \mathrm{Cl}) \\
\text { (SE) }\end{array}$} & \multirow[b]{2}{*}{ D } & & & & & & & \\
\hline & & B & C & & \multirow[t]{2}{*}{ Wald F } & \multirow[t]{2}{*}{ df1 } & \multirow[t]{2}{*}{ df2 } & \multirow[t]{2}{*}{$\mathbf{p}$} & \multirow[t]{2}{*}{ D/A } & \multirow[t]{2}{*}{ CIA } & \\
\hline & & $N=4157$ & $N=1802$ & $N=88$ & & & & & & & \\
\hline QUALITY OF LIFE INDEX & & & & & & & & & & & \\
\hline $\begin{array}{l}\text { Total Score }(7.45,95 \% \\
\text { IC } 7.39-7.50)\end{array}$ & $\begin{array}{c}7.82 \\
(\mathrm{SE} \mathrm{0.07)} \\
(7.69-7.95)\end{array}$ & $\begin{array}{c}7.50 \\
(\mathrm{SE} 0.03) \\
(7.45-7.56)\end{array}$ & $\begin{array}{c}7.18 \\
(\mathrm{SE} 0.05) \\
(7.08-7.28)\end{array}$ & $\begin{array}{c}6.81 \\
(\text { SE 0.20) } \\
(6.41-7.21)\end{array}$ & 5.628 & 3 & 578 & $<0.0001$ & $<0.0001$ & $<0.001$ & $<0.001$ \\
\hline $\begin{array}{l}\text { Physical Wellbeing } \\
(7.04,95 \% \text { IC } 6.97- \\
7.10)\end{array}$ & $\begin{array}{c}7.33 \\
(\mathrm{SE} 0.14) \\
(7.05-7.60)\end{array}$ & $\begin{array}{c}7.13 \\
(\mathrm{SE} 0.04) \\
(7.06-7.20)\end{array}$ & $\begin{array}{c}6.71 \\
(\mathrm{SE} 0.09) \\
(6.54-6.88)\end{array}$ & $\begin{array}{c}6.23 \\
(\mathrm{SE} 0.22) \\
(5.80-6.67)\end{array}$ & 13.909 & 3 & 578 & $<0.0001$ & 0.00004 & 0.00006 & 0.195 \\
\hline $\begin{array}{l}\text { Emotional Wellbeing } \\
\text { (7.50, 95\% IC } 7.43- \\
7.58)\end{array}$ & $\begin{array}{c}7.94 \\
(\mathrm{SE} 0.10) \\
(7.74-8.13)\end{array}$ & $\begin{array}{c}7.54 \\
(\mathrm{SE} 0.04) \\
(7.47-7.61)\end{array}$ & $\begin{array}{c}7.27 \\
(\mathrm{SE} 0.11) \\
(7.06-7.49)\end{array}$ & $\begin{array}{c}6.92 \\
(\mathrm{SE} 0.26) \\
(6.42-7.42)\end{array}$ & 9.969 & 3 & 578 & $<0.0001$ & 0.0002 & $<0.0001$ & 0.0002 \\
\hline $\begin{array}{l}\text { Self care }(8.15,95 \% \\
\text { IC } 8.08-8.23)\end{array}$ & $\begin{array}{c}8.53 \\
(\mathrm{SE} 0.10) \\
(8.34-8.71)\end{array}$ & $\begin{array}{c}8.19 \\
(\mathrm{SE} \mathrm{0.04)} \\
(8.10-8.27)\end{array}$ & $\begin{array}{c}7.95 \\
(\mathrm{SE} 0.06) \\
(7.83-8.07)\end{array}$ & $\begin{array}{c}7.62 \\
(\mathrm{SE} \mathrm{0.28)} \\
(7.07-8.16)\end{array}$ & 10.503 & 3 & 578 & $<0.0001$ & 0.002 & $<0.0001$ & 0.002 \\
\hline $\begin{array}{l}\text { Occupational } \\
\text { Functioning (8.00, 95\% } \\
\text { IC 7.92-8.07) }\end{array}$ & $\begin{array}{c}8.41 \\
(\mathrm{SE} 0.09) \\
(8.23-8.58)\end{array}$ & $\begin{array}{c}8.06 \\
(\mathrm{SE} 0.05) \\
(7.97-8.14)\end{array}$ & $\begin{array}{c}7.72 \\
\text { (SE 0.08) } \\
(7.57-7.87)\end{array}$ & $\begin{array}{c}7.17 \\
(\mathrm{SE} 0.26) \\
(6.66-7.69)\end{array}$ & 14.332 & 3 & 578 & $<0.0001$ & 0.00001 & $<0.0001$ & 0.001 \\
\hline $\begin{array}{l}\text { Interpersonal } \\
\text { Functioning (7.99, } \\
95 \% \text { IC 7.92-8.05) }\end{array}$ & $\begin{array}{c}8.27 \\
(\mathrm{SE} \mathrm{0.10)} \\
(8.08-8.47)\end{array}$ & $\begin{array}{c}8.04 \\
(\mathrm{SE} 0.04) \\
(7.97-8.11)\end{array}$ & $\begin{array}{c}7.76 \\
(\mathrm{SE} 0.07) \\
(7.63-7.90)\end{array}$ & $\begin{array}{c}7.36 \\
(\mathrm{SE} 0.33) \\
(6.72-8.00)\end{array}$ & 6.815 & 3 & 578 & 0.0002 & 0.008 & 0.0001 & 0.02 \\
\hline $\begin{array}{l}\text { Social support }(7.35 \text {, } \\
95 \% \text { IC } 7.26-7.45)\end{array}$ & $\begin{array}{c}7.89 \\
(\mathrm{SE} \mathrm{0.15)} \\
(7.59-8.18)\end{array}$ & $\begin{array}{c}7.42 \\
(\mathrm{SE} 0.05) \\
(7.33-7.52)\end{array}$ & $\begin{array}{c}6.99 \\
(\mathrm{SE} 0.07) \\
(6.85-7.13)\end{array}$ & $\begin{array}{c}6.65 \\
(\mathrm{SE} \mathrm{0.22)} \\
(6.23-7.07)\end{array}$ & 20.385 & 3 & 578 & $<0.0001$ & $<0.0001$ & $<0.0001$ & 0.002 \\
\hline $\begin{array}{l}\text { Communitarian } \\
\text { support (5.65, 95\% IC } \\
5.57-5.79)\end{array}$ & $\begin{array}{c}6.12 \\
(\mathrm{SE} 0.21) \\
(5.71-6.53)\end{array}$ & $\begin{array}{c}5.68 \\
(\mathrm{SE} 0.06) \\
(5.56-5.79)\end{array}$ & $\begin{array}{c}5.53 \\
(\mathrm{SE} 0.09) \\
(5.36-5.71)\end{array}$ & $\begin{array}{c}5.49 \\
(\mathrm{SE} 0.31) \\
(4.88-6.11)\end{array}$ & 2.845 & 3 & 578 & 0.037 & 0.101 & 0.005 & 0.03 \\
\hline $\begin{array}{l}\text { Personal fulfillment } \\
\text { (7.36, 95\% IC7.28- } \\
7.44)\end{array}$ & $\begin{array}{c}7.79 \\
(\mathrm{SE} 0.08) \\
(7.63-7.96)\end{array}$ & $\begin{array}{c}7.43 \\
(\mathrm{SE} 0.04) \\
(7.35-7.51)\end{array}$ & $\begin{array}{c}7.03 \\
(\mathrm{SE} 0.07) \\
(6.89-7.18)\end{array}$ & $\begin{array}{c}6.93 \\
(\mathrm{SE} \mathrm{0.34)} \\
(6.27-7.59)\end{array}$ & 17.304 & 3 & 578 & $<0.0001$ & 0.014 & $<0.0001$ & 0.00005 \\
\hline $\begin{array}{l}\text { Spiritual satisfaction } \\
(7.67,95 \% \text { IC } 7.60- \\
7.73)\end{array}$ & $\begin{array}{c}7.84 \\
(\mathrm{SE} 0.13) \\
(7.59-8.10)\end{array}$ & $\begin{array}{c}7.72 \\
(\text { SE0.04) } \\
(7.65-7.79)\end{array}$ & $\begin{array}{c}7.47 \\
(\text { SE } 0.07) \\
(7.34-7.60)\end{array}$ & $\begin{array}{c}7.28 \\
(\mathrm{SE} \mathrm{0.25)} \\
(6.80-7.76)\end{array}$ & 5.117 & 3 & 578 & 0.002 & 0.044 & 0.005 & 0.338 \\
\hline $\begin{array}{l}\text { Global Quality of Life } \\
(7.75,95 \% \text { IC } 7.69- \\
7.81)\end{array}$ & $\begin{array}{c}8.07 \\
(\mathrm{SE} \mathrm{0.10)} \\
(7.88-8.27)\end{array}$ & $\begin{array}{c}7.84 \\
(\mathrm{SE} \mathrm{0.03)} \\
(7.77-7.90)\end{array}$ & $\begin{array}{c}7.44 \\
(\mathrm{SE} 0.08) \\
(7.29-7.59)\end{array}$ & $\begin{array}{c}6.66 \\
(\mathrm{SE} \mathrm{0.27)} \\
(6.13-7.19)\end{array}$ & 15.057 & 3 & 578 & $<0.0001$ & $<0.0001$ & $<0.001$ & 0.027 \\
\hline
\end{tabular}

$\left(^{*}\right) \mathrm{A}=$ Cover the basic needs and other needs (Not poor); $\mathrm{B}=$ Cover the basic needs but not others (Just not poor); C= Cover only basic nutritional needs (Poor); $\mathrm{D}=$ Do not even cover the basic nutritional needs (Extremely Poor).

\section{Discussion}

This study contributes to the ongoing literature on the subject by demonstrating an association of positive mental health indicators and poverty indicators. In fact, almost all indicators used in this study were significantly in the direction of worst rates in individuals living in poverty conditions. No previously studies of this type have been conducted in developing countries and other contributions have focus on other areas. Feelings of happiness were less prevalent $(p<0.046)$ in individuals in poverty compared to those "not poor". Socioeconomic factors have been related to happiness in regard to income [35], but some studies yield contradictory findings. One explanation for this was suggested in a study conducted in the United States and 


\section{Table 7. Prevalence of disability in the population in five cities of the coast of Peru $(n=6,555)$ according to the Perceived Coverage of Basic Needs with Family Income and Prevalence Ratio Among the Poor and Not Poor}

\begin{tabular}{|c|c|c|c|c|c|c|c|c|c|c|c|}
\hline \multirow{3}{*}{$\begin{array}{c}\text { Prevalences of } \\
\text { positive mental health } \\
\text { indicators (General } \\
\text { Population) }\end{array}$} & \multicolumn{4}{|c|}{ Coverage of Basic Needs with Family Income $\left({ }^{*}\right)$} & \multirow{2}{*}{\multicolumn{4}{|c|}{ Independence Test }} & \multirow{2}{*}{\multicolumn{3}{|c|}{ Prevalence Ratio }} \\
\hline & \multicolumn{4}{|c|}{$\begin{array}{c}(95 \% \mathrm{Cl}) \\
(\mathrm{SE})\end{array}$} & & & & & & & \\
\hline & $\underset{N=501}{A}$ & $\begin{array}{c}\text { B } \\
N=4157\end{array}$ & $\begin{array}{c}\mathbf{C} \\
\mathrm{N}=1802\end{array}$ & $\begin{array}{c}D \\
N=88\end{array}$ & Wald F & df1 & df2 & $\mathbf{p}$ & $\mathrm{B} / \mathrm{A}$ & ClA & D/A \\
\hline $\begin{array}{l}\text { FUNCTIONING } \\
\text { PROBLEMS } \\
\text { (AT LEAST SOME } \\
\text { DISABILITY) }\end{array}$ & & & & & & & & & & & \\
\hline $\begin{array}{l}\text { Any disability (10.7, } \\
95 \% \text { IC } 9.3-12.4)\end{array}$ & $\begin{array}{c}8.5 \\
(5.5-13.1)\end{array}$ & $\begin{array}{c}10.4 \\
(8.7-12.3)\end{array}$ & $\begin{array}{c}12.2 \\
(9.7-15.3)\end{array}$ & $\begin{array}{c}17.8 \\
(8.8-32.6)\end{array}$ & 1.522 & 2.836 & 1645 & 0.209 & 1.22 & 1.44 & 2.09 \\
\hline $\begin{array}{l}\text { Self-care }(1.6 \%, 95 \% \\
\text { IC 1.1-2.3) }\end{array}$ & $\begin{array}{c}0.2 \\
(0.0-0.6)\end{array}$ & $\begin{array}{c}1.4 \\
(0.9-2.3)\end{array}$ & $\begin{array}{c}2.4 \\
(1.4-4.0)\end{array}$ & $\begin{array}{c}8.5 \\
(2.5-25.1)\end{array}$ & 5.703 & 2.197 & 1274 & 0.002 & 7.0 & 12.0 & 42.5 \\
\hline $\begin{array}{l}\text { Occupational } \\
\text { functioning (5.2\%., 95\% } \\
\text { IC } 4.3-6.4)\end{array}$ & $\begin{array}{c}2.8 \\
(1.4-5.4)\end{array}$ & $\begin{array}{c}5.1 \\
(4.0-6.6)\end{array}$ & $\begin{array}{c}6.1 \\
(4.6-8.2)\end{array}$ & $\begin{array}{c}12.3 \\
(5.1-26.9)\end{array}$ & 2.610 & 2.867 & 1663 & 0.053 & 1.82 & 2.18 & 4.39 \\
\hline $\begin{array}{l}\text { Family functioning } \\
(3.6 \%, 95 \% \text { IC } 2.9-4.4)\end{array}$ & $\begin{array}{c}3.4 \\
(1.7-6.7)\end{array}$ & $\begin{array}{c}3.2 \\
(2.5-4.2)\end{array}$ & $\begin{array}{c}4.2 \\
(2.9-6.0)\end{array}$ & $\begin{array}{c}12.3 \\
(4.6-29.0)\end{array}$ & 2.527 & 2.953 & 1713 & 0.057 & 0.94 & 1.24 & 3.61 \\
\hline $\begin{array}{l}\text { Broad Social } \\
\text { Functioning (3.2, 95\% } \\
\text { IC 2.5-4.1) }\end{array}$ & $\begin{array}{c}2.0 \\
(0.8-4.8)\end{array}$ & $\begin{array}{c}3.1 \\
(2.4-4.1)\end{array}$ & $\begin{array}{c}3.7 \\
(2.4-5.6)\end{array}$ & $\begin{array}{c}10.9 \\
(3.9-26.7)\end{array}$ & 2.237 & 2.930 & 1700 & 0.084 & 1.55 & 1.85 & 5.45 \\
\hline $\begin{array}{l}\text { Organizational } \\
\text { Functioning (3.9, 95\% } \\
\text { IC 3.2-4.7) }\end{array}$ & $\begin{array}{c}3.5 \\
(1.8-6.5)\end{array}$ & $\begin{array}{c}3.7 \\
(2.9-4.7)\end{array}$ & $\begin{array}{c}4.5 \\
(3.3-6.1)\end{array}$ & $\begin{array}{c}8.2 \\
(2.3-25.2)\end{array}$ & 0.887 & 2.982 & 1730 & 0.447 & 1.06 & 1.29 & 2.34 \\
\hline
\end{tabular}

$\left(^{*}\right) A=$ Cover the basic needs and other needs (Not poor); $B=$ Cover the basic needs but not others (Just not poor); C= Cover only basic nutritional needs (Poor); D= Do not even cover the basic nutritional needs (Extremely Poor).

eight developed countries which confirms that the range and skew of the income distribution in a community affects a person's happiness, supporting the effect of social comparison of income within a community on happiness [36]. In this sense, happiness would depend on the welfare of the neighbor and not on the limitations encountered in the proper family. Most of these studies are conducted in places where the satisfaction of basic need is not an issue. For developing countries, not only could the social comparison effect matter, but also the direct limitations that are encountered in a daily basis, which in our study are related to happiness.

Our study found significantly lower level of personal satisfaction in regard to physical aspect, skin color, intelligence, socioeconomic status, profession studied, level of achieved education, social relationships and place of residence among the poor individuals compared with persons with no problems in meeting the basic needs with household income. No studies were found that specifically address personal satisfaction in these areas. Many of these conditions give rise to reflections of social exclusion and opportunities, thereby implying the presence of full inequality to access essential rights by the poor people [37]. One recent meta-analytic study has found a medium to strong relation between socioeconomic status andacademic achievement that could be related to dissatisfaction with the level of achieved education [38].
In regard to family relationships, all measures were highly significant, showing lower frequencies of good family interactions in poor individuals compared to not poor subjects. Poverty have been related to circumstances, such as the separation of the family, a decrease in the abilities of parents in their role towards their children due to environments charged with insecurity and stress, an increase in the affliction of the parents that disturbs the relationship with their children, parental depression, reduction of the parents' power in the case of children, one parent families, inefficient upbringing and family dysfunction [39,40,41,42]. Children from lower socioeconomic status are more prone to have unresponsive and harsher attitudes from their parents affecting their relationships [43].

Our study found greater level of psychosocial stressors among the poor individuals compared with not poor ones. This findings were significant for tension or problems perceived related to work $(\mathrm{p}=0.03)$, children or other relatives $(<0.0001)$, money $(\mathrm{p}<0.0001)$ and health $(p=0.001)$. Households living in poverty have been shown to have more financial pressure and cumulative, multiple stressors than middle-income counterparts, particularly in children [44].

More poor individuals in our study present higher disturbances in regard to smell, dust and garbage than not poor subjects. No significant differences were found for noise and lack of space. Other studies have related noise and crowding to poverty [44]. Environmental stressors 
have been related to increase in the measures of hemodynamic, endocrine, and neuroendocrine values and decreased performance on complex tasks, and they reported a subjective increase in stress, pointing out the importance of environmental stressors to health [45].

In regard to quality of life as measured by the Quality of Life Index, lower levels on all aspects of quality of life were found in the poor individual compared to not poor persons. Differences were found in most cases between both poor levels and not poor levels. Health related quality of life (HRQL) studies regarding mobility, selfcare, usual activities, pain/discomfort, and anxiety/depression found lower HRQL scores associated with race/ethnicity, income and education [46]. Another study conducted in European countries in older people has demonstrated that quality of life was associated with socioeconomic position and education, but no specific quality of life aspect was reported [47]. A study in a sample of 2065 subjects in Norway found that income was correlated with Quality of life measures [48]. In this study aspects of quality of live statistically significant were subjective wellbeing, self-realization and absence of negative life events. Not statistically significant were neighborhood quality, contact with friends, support if ill and contact with family of origin. In our study, socioeconomic status was measured with a question regarding the coverage of basic needs with household income a not household income itself. Income as has been noted by a previously mention study the range and skew of the income distribution in a community affects a person's happiness [36], and therefore could influence quality of life measures. Poor persons identified in our study could correspond to individuals with more serious economic conditions.

With respect to functioning our study found greater level of disability regarding self care in poor people as compared with not poor individuals. Poor people's disabilities have been related to malnutrition and poor health care, aspects related to self-care [49]. Also sustained economic hardship has been associated with difficulties with independent activities of daily living (cooking, shopping, and managing money) and activities of daily living (such as walking, eating, dressing, and using the toilet); mean age for this group was far higher than ours (63.4-65.2 years) [50] but our findings could reflect a tendency. We did not find studies relating specifically the areas of adaptive functioning suggested by WHO and socioeconomic status or poverty. Other functioning areas in our study were worst in poor people but did not reach statistically differences. It is possible that a bigger sample could yield better results. Disability has been proposed to be related to poverty in interaction with sense of coherence, environmental risk factors, social role devaluation and group membership factors [51].

The findings of this study must be considered in the light of the following limitations: First, given that it is a survey related to mental health, systematic negative reports cannot be ruled out either because the persons were consciously unwilling to answer out of fear or stigmatization. Second, a large part of the survey relies on the perspective and subjective opinion of the surveyed persons which could lead to bias. Third, the results cannot be generalized to the rest of the country because of multicultural issues and reports that suggest the existence of this variability [52]. Fourth, some issues demand a bigger sample size. Finally, further longitudinal studies are encouraged in this issue of positive mental health in order to overcome the limitations of the cross-sectional method.

Nevertheless, many of our findings bring into attention the problem of social exclusion and its relation to poverty [49]. Person centered approaches to medicine and mental health give the opportunity to enhance health policies directed to less favorable people by paying attention to various aspects of the person such as his quality of life, personal satisfaction, personal environmental problems, daily stressor and disabilities. Implications of socioeconomic aspects in regard to the comprehensive diagnostic processes, treatment and research, particularly in less-developed countries should be considered. Health policies based on person centered care programs through the assessment of positive health could help to be more sensitive to more vulnerable sectors of the populations.

\section{References}

[1] Stewart M. (2001). Towards a global definition of patient centred care. The patient should be the judge of patient centr ed care. British Medical Journal 322:444-5.

[2] Little P, Everitt H, Williamson I, Warner G, Moore M, Gould C, Ferrier K, Payne S. (2001). Observational study of effect of patient centredness and positive approach on outcomes of general practice consultations. British Medical Journal ;323:908-11.

[3] Tauber AI. (2005). Medicine and the Call for a Moral Epistemology. Perspective in Biology and Medicine 48(1): 42-53. [4] Hughes JC, Bamford C, May C. (2008). Types of centredness in health care: themes and concepts. Medical Health care and Philosophy 11:455-463.

[5] Mezzich JE, Salloum IM. (2007). Towards innovative international classification and diagnostic systems: ICD-11 and person-centered integrative diagnosis. Acta Psychiatrica Scandinavica 116: 1-5

[6] Mezzich JE. (2005). Positive Health: Conceptual Place, Dimensions and Implications. Psychopathology 38:177-179.

[7] Korkeila JA. (2000). Measuring Aspects of Mental Health. Helsinky: Stakes.

[8] Cloninger R: (2002). Implications of comorbidity for the classifications of mental disorders: The need for a psychobiology of coherence; in Maj M, Gaebel W, et al (eds): Psychiatric Diagnosis and Classification. Chichester, Wiley.

[9] Herrman H, Saxena S Moodie R (eds): (2005). Promoting Mental Health: Concepts, Emerging Evidence, Practice. Geneva, WHO.

[10] Magyary D. (2002). Positive Mental health: a turn of the century perspective. Issues in Mental Health Nursing 23: 331349.

The International Journal of Person Centered Medicine Volume 1 Issue 1 pp 62-75 
[11] Schmolke M, Lecic-Tosevsky D (eds): (2003). Health promotion: An integral component of effective clinical care. Dynamic Psychiatry 36 (special issue):221-319.

[12] Ramírez, E. (1989). Sistema Octogonal de Evaluación Psiquiátrica Technical Report. San Juan, Puerto Rico: Secretaría de Salud Mental.

[13] Otero Ojeda, A, A. (Ed.) . (2000). Tercer Glosario Cubano de Psiquiatría. La Habana: Hospital Psiquiátrico de La Habana.

[14] Mezzich JE, Kleinman A, Fabrega H, Parron DL. (1996) Culture and Psychiatric Diagnosis: A DSM-IV Perspective. Washington, D.C.: American Psychiatric Press.

[15] WPA IGDA Workgroup,. IGDA.7. (2003). Standardized multi-axial diagnostic formulation. British Journal of Psychiatry 182 (supp. 45): s52-s54.

[16] WPA IGDA Workgroup. IGDA. 8. (2003). Idiographic (personalized) diagnostic formulation. British Journal of Psychiatry 182 (supp. 45): s55-s57

[17] Sección de Diagnóstico y Clasificación de la Asociación Psiquiátrica de América Latina. Guía Latinoamericana de Diagnóstico Psiquiátrico. Guadalajara: Asociación Psiquiátrica de América Latina; 2004.

[18] Corin E, Bibeau G, Martin JC \& Laplante R. Comprendre pour agir autrement: repères pour régionaliser les services de santé mentale. Montréal, Québec (Canada): Presses de l'Université de Montréal, 1990.

[19] Murray S. (2006). Poverty and health. Canadian Medical Association Journal 174(7): 923.

[20] CEPAL: Comisión Económica para América Latina y el Caribe: División de Estadística y Proyecciones Económicas. Unidad de Estadísticas Sociales, sobre la base de tabulaciones especiales de las encuestas de hogares de los respectivos países. http://websie.eclac.cl/sisgen/ConsultaIntegrada.asp?idAplicacion =14\&idioma=e. Accessed 22 April 2010.

[21] Desjarlais R, Eisenberg L, Good B, Kleinman A. (1997). Salud Mental en el Mundo: Problemas y Prioridades en Poblaciones de Bajos Ingresos. Washington: OPS-OMS; p. 1-31. [22] Salganicoff A, Wyn R. (1999). Access to Care for LowIncome Women: The impact of Medicaid. Journal of Health care for the Poor and Underserved 10(4): 453-467.

[23] Instituto Nacional de Salud Mental "Honorio Delgado Hideyo Noguchi”. Estudio Epidemiológico de Salud Mental en la Costa Peruana 2006. Anales de Salud Mental 2007; XXIII(1-2):1228.

[24] Instituto Especializado de Salud Mental "Honorio Delgado Hideyo Noguchi”. Estudio Epidemiológico Metropolitano de Salud Mental 2002. Informe General. Anales de Salud Mental 2002; XVIII (1-2): 1-197

[25] Instituto Especializado de Salud Mental "Honorio Delgado Hideyo Noguchi”. Estudio Epidemiológico de Salud Mental en la Sierra Peruana 2003. Informe General. Anales de Salud Mental 2004; XIX (1-2): 1-209

[26] Instituto Especializado de Salud Mental "Honorio Delgado Hideyo Noguchi” Estudio Epidemiológico en Salud Mental en la Selva Peruana. Anales de Salud Mental 2005; XXI (1-2): 1-212. [27] Instituto Nacional de Salud Mental. Estudio Epidemiológico de Salud Mental en Fronteras 2005. Informe General. Anales de Salud Mental 2006; XXII (1-2): 1-227

[28] Instituto Nacional de Salud Mental. Confiabilidad y validez de los cuestionarios de salud mental de Lima y de la Selva Peruana. Anales de Salud Mental 2009; XXV (Supl. 1): 1-259.

[29] Ministerio de Salud. Estudio Nacional de Salud Mental y Consumo de Sustancias Psicoactivas. Editorial Carrera Séptima. Santa Fe de Bogotá, 1993.
[30] Mezzich JE, Ruipérez MA, Pérez C, Ion G, Liu J, Mamad S. (2000). The Spanish version of the quality of life index: presentation and validation. Journal of Nervous and Mental Disease 188:301-305.

[31] World Health Organization (1997). Multiaxial Presentation of ICD-10 for Adults. Cambridge, U.K.:

Cambridge University Press.

[32] Perales A, Sogi C, Sánchez E, Salas RE. (1995). Salud Mental de una población urbano marginal de Lima, en Serie: Monografías de Investigación No 2. Lima: INSM Honorio Delgado- Hideyo Noguchi”.

[33] Schwartz KI, Zapata-Vega M I, Mezzich JE, Mazzotti G. (2006). Validation study of the Multicultural Quality of Life Index (MQLI) in a Peruvian sample. Revista Brasileira de Psiquiatria 28(1):24-8.

[34] World Health Organization (1997). Multiaxial Presentation of ICD-10 for Adults. Cambridge, U.K.: Cambridge University Press.

[35] Easterlin RA. (2003). Explaning happiness. Proceedings of the National Academy of Sciences of the United States of America 100(19):11176-11183.

[36] Hagerty MR. (2000). Social comparisons of income in one's community: evidence from national surveys of income and happiness. Journal of Personality and Social Psychology 78(4):764-771.

[37] OPS-CEPAL. Salud, Equidad y Transformación Productiva en América Latina y el Caribe. Cuaderno Técnico No 46. OPS; 1997. p. 45

[38] Sirin SR. (2005). Socioeconomic Status and Academic Achievement: A Meta-Analytic Review of Research. Review of Educational Research 75(3):417-453.

[39] Beiser M, Hou F, Imán 1, Tousignant M. Poverty, (2002). Family Process, and the Mental Health of Immigrant Children in Canada. American Journal of Public Health Feb; 92(2):220-7.

[40] Robichaud JB, Guay L., Colin C, Pothier M. (1994). Les liens entre la pauvrété et la santé mentale: de l éxcusion á léquite. Montreal: Gaetan Morin Editeur; p. 93-98.

[41] U.S. Census Bureau. (2000). Population survey (March Suppl. 2000). Washington, DC: U.S. Government Printing Office.

[42] Evans GW. (2004). The environment of Childhood Poverty. American Psychologist 59(2): 77-92.

[43] Bradley, R. H., Corwyn, R. F., McAdoo, H., \& Coll, C. (2001). The home environments of children in the United States: Part I. Variations by age, ethnicity, and poverty status. Child Development 72(6): 1844-1867.

[44] Evans GW, English K. (2002) The Environment of Poverty: Multiple Stressor Exposure, Psychophysiological Stress, and Socioemotional Adjustment. Child Development 73(4): 12381248.

[45] Martimportugués-Goyenechea C, Gómez-Jacinto L. (2005). Simultaneous multiple stressors in the environment: physiological stress reactions, performance, and stress evaluation. Psychological Report 97(3):867-74.

[46] Lubetkin EI, JiaH, Franks P, Gold MR.*2005). Relationship among sociodemographic factors, clinical conditions, and healthrelated quality of life: Examining the EQ-5D in the U.S. general population. Quality of Life Research (14): 2187-2196.

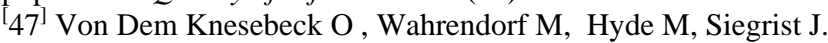
(2007). Socio-economic position and quality of life among older people in 10 European countries: results of the SHARE study. Ageing \& Society (27): 269-284. 
[48] Cramer V, Torgersen S, Kringlen E. (2007). SocioDemographic Conditions, Subjective Somatic Health, Axis I Disorders and Personality Disorders in the Common Population: the Relationship to Quality of Life. Journal of Personality Disorders 21( 5): 552-567.

[49] Department for International Development. Reducing poverty by tackling social exclusion: a DFID policy paper. September, 2005. http://www.dfid.gov.uk/Documents/publications/socialexclusion.pdf (accessed April 21,2010).

[50] Lynch JW, Kaplan GA, Shema SJ. (1997). Cumulative Impact Of Sustained Economic Hardship On Physical, Cognitive, Psychological, And Social Functioning. The New England Journal of Medicine 337:1889-95

[51] Daniel C Lustig; David R Strauser. Causal Relationships (2007). Between Poverty and Disability. Rehabilitation Counseling Bulletin. 50 (4): 194-202.

[52] Costello EJ, Messer SC, Bird HR. Cohen P, Reinherz HZ. (1998). The Prevalence of Serious Emotional Disturbance: a ReAnalysis of Community Studies. Journal of Child and Family Studies 7: 411-432. 